\title{
Participo, me cuido y mejoro mi salud: plan de acción personalizado
}

\author{
Participo, me cuido y mejoro mi salud con el apoyo de los profesionales \\ que me atienden: mi plan de acción personalizado y el uso \\ de las tecnologías como apoyo al proceso asistencial
}

Ante la situación de pandemia generada por la infección de COVID-19, las actuaciones de los sistemas sanitarios se han centrado, principalmente, en garantizar una asistencia sanitaria específica a las necesidades creadas por esta enfermedad, incorporando medidas vinculadas a la prevención y a la protección de la salud. Esto ha provocado una reordenación de la oferta asistencial, que ha afectado a los procesos de atención en general y, en concreto, a la atención a las personas con enfermedades crónicas. Las personas con patologías crónicas de alta complejidad clínica y/o asistencial han visto mermadas en intensidad y calidad las actuaciones previamente priorizadas desde los sistemas sanitarios.

En períodos previos a la aparición de la pandemia, los sistemas de salud habían puesto énfasis en adaptar recursos y planificar medidas orientadas a ofrecer una atención integral a las personas con enfermedades crónicas, dando mayor protagonismo a la Atención Primaria (AP), poniendo en marcha herramientas para facilitar la accesibilidad a los profesionales de referencia, y potenciando la continuidad asistencial y el autocuidado'.

Para favorecer la respuesta a la ciudadanía en estos casos de mayor complejidad, se ha impulsado el desarrollo de nuevas competencias profesionales, así como la creación de herramientas digitales que permiten la actuación conjunta y coordinada del equipo de profesionales de referencia (médico y enfermera de AP, Enfermera Gestora de Casos, médico de atención hospitalaria, especialmente Medicina Interna como referente en estos procesos de cronicidad compleja, y otros profesionales) y la participación del paciente y su familia en su propia salud y autocuidado ${ }^{2-4}$.

En el Servicio Andaluz de Salud, estas nuevas herramientas se concretaron en el diseño, aplicación y extensión de los Planes de Acción Personalizados (PAP). Los PAP permiten planificar la asistencia sanitaria, coordinan las actuaciones del equipo profesionales y permiten la implicación del paciente, familia y/o personas que cuidan, para que se sientan verdaderamente el centro de la atención sanitaria ${ }^{5}$.

Los PAP, además de permitir esta planificación estructurada de las actuaciones de los profesionales que intervienen en torno al caso, se componen también de las recomendaciones dadas al paciente y/o su familia para el autocuidado (mi plan de acción personalizado). Estas recomendaciones deben estar accesibles para los pacientes y/o familia y, al menos, entregarlas $y$ explicarlas para que se puedan llevar a cabo, quedando siempre el profesional a disposición del paciente para resolver dudas y acompañar en procesos de aprendizaje o refuerzos.

Además de la atención a personas con enfermedades crónicas complejas, para poder prevenir las secuelas y el exceso de mortalidad añadido por las dificultades de atención a la cronicidad', en la medida de lo posible, se ha de continuar con la atención de las personas con mayor complejidad tanto de forma presencial como en el domicilio. Para ello, la utilización de las nuevas tecnologías resulta imprescindible en la realización de seguimientos proactivos domiciliarios, sobre todo ahora que la presencialidad en la pandemia ha quedado mermada. En este sentido, la realización de llamadas telefónicas estructuradas ha tenido un especial protagonismo durante la pandemia, y en un futuro a medio o largo plazo podría evolucionar a sistemas de videollamadas.

Estas herramientas han de prepararse como soporte para que, tras el seguimiento, a través de un cuestionario estructurado, permitan tomar decisiones que den continuidad a la atención. De esta forma, se facilita a los profesionales la captación proactiva de pacientes y la valoración de su situación actual, para poder reordenar el posterior seguimiento programado telemático o presencial de los mismos. Con el uso de estas herramientas de soporte se mejorará progresivamente la accesibilidad y el seguimiento programado de las situaciones clínicas con el objetivo de: anticipar problemas de salud a través de la recogida de información sobre signos y síntomas; detectar alertas clínicas; evitar o disminuir la polimedicación; explorar la adherencia al tratamiento; valorar la capacidad de autocuidados y de mejora de calidad de vida; facilitar un mayor apoyo a los cuidadores/as; detectar el riesgo social y prevenir futuros casos de COVID-19 y sus secuelas.

Existen sistemas de registros, como el desarrollado por el Servicio Andaluz de Salud, que se han diseñado como herramienta inteligente, integrada en la historia de salud digital del paciente que permite la realización de cada una de las partes de las que se compone el PAP: la valoración integral (VIE), intervenciones de cada profesional y recomendaciones asociadas. Este sistema inteligente permite relacionar los ítems alterados de la valoración con las intervenciones y recomendaciones que se podrán desarrollar con los pacientes y/o familia.

Además, y para facilitar la accesibilidad con el ámbito hospitalario y la coordinación entre los profesionales referentes de estos pacientes (coordinación de inicio y/o seguimiento y sobre todo ante desestabilización o complicaciones), se utilizan las nuevas tecnologías para desarrollar servicios de teleconsulta, generalmente, en este tipo de pacientes, entre el equipo de AP y el servicio de Medicina Interna hospitalaria u otras especialidades, cuando sea necesario.

Por último, nos gustaría destacar que, a pesar del importante papel que juegan las nuevas herramientas en la planificación y coordinación de la asistencia sanitaria, resulta imprescindible que los equipos de profesionales impliquen, de forma participativa, a pacientes y familias en sus procesos de salud y enfermedad. Cada vez más pacientes quieren autocuidarse y tomar las riendas de su salud, ¿estamos dispuestos a acompañarles? 
Nieves Lafuente-Robles

Directora de la Estrategia de Cuidados de Andalucía Servicio Andaluz de Salud. Consejería de Salud y Familias. Sevilla. España

Nieves.lafuente.sppa@juntadeandalucia.es

Citar como: Lafuente-Robles N. Participo, me cuido y mejoro mi salud: plan de acción personalizado. Rev Esp Casos Clin Med Intern (RECCMI). 2021 (abril); 6(1): 1-2. doi:10.32818/reccmi.a6n1a1.

Cite this as: Lafuente-Robles N. I participate, take care of myself and improve my health: personalized action plan. Rev Esp Casos Clin Med Intern (RECCMI). 2021 (April); 6(1): 1-2. doi: 10.32818/reccmi.a6n1a1.

\section{Bibliografía}

1. Consejería de Salud. Junta de Andalucía. Plan Andaluz de Atención Integrada a pacientes con enfermedades crónicas 2012/ 2016. Sevilla 2012. Disponible en: https://www.juntadeandalucia.es/export/drupaljda/salud_5af06533c7e30_PIEC.pdf (Consultado: 30 marzo 2021).

2. Sánchez-Martín Cl. Cronicidad y complejidad: nuevos roles en Enfermería. Enfermeras de Práctica Avanzada y paciente crónico. Enferm Clin. 2014; 24(1): 79-89. Spanish. doi: 10.1016/j.enfcli.2013.12.007.

3. Coulter A, Entwistle VA, Eccles A, Ryan S, Shepperd S, Perera R. Personalised care planning for adults with chronic or long-term health conditions. Cochrane Database Syst Rev. 2015; 2015(3): CD010523. doi: 10.1002/14651858. CD010523.pub2
4. The NHS Long Term Plan. National Health Service. England. January 2019. Accesible en: https://www.longtermplan.nhs.uk. (último acceso abril 2021).

5. Servicio Andaluz de Salud. Consejería de Salud. Junta de Andalucía. Atención al paciente crónico con necesidades de salud complejas. Sevilla 2016. Disponible en: https://www.sspa.juntadeandalucia.es/servicioandaluzdesalud/agsemaxarquia/wp-content/uploads/Plan-Atenci\%C3\%B3n-Pacientes-Cr\%C3\%B3nicos.pdf (último acceso 30 marzo 2021).

6. Recomendaciones para abordaje de la fragilidad en situación de crisis sanitaria generada por la covid-19. Grupo de trabajo de prevención de fragilidad y caídas en la persona mayor. Ministerio de Sanidad. Gobierno de España. Madrid, marzo 2021. 